\title{
Optimum Rate of Blended Fertilizer and Urea Determination for Potato Production under Irrigation Condition in Holetta Research Center, West Showa, Ethiopia
}

\author{
Egata Shunka, ${ }^{1, *}$, Abebe Chindi ${ }^{1}$, Kassaye Negash ${ }^{1}$, Gebremedhin W/Giorigis ${ }^{1}$, Tesfaye Abebe ${ }^{1}$, Atsede \\ Solomon $^{1}$, Mihiretu Bedasa ${ }^{2}$, Ibrahim Said ${ }^{1}$, Lema Tesema ${ }^{1}$ \\ ${ }^{1}$ Ethiopian Institute of Agricultural Research, Holetta Agricultural Research Center, Crop Research Process, Potato Research Pro- \\ gram, Addis Ababa, Ethiopia. \\ ${ }^{2}$ Ethiopian Institute of Agricultural Research, Holetta Agricultural Research Center, Natural Resource Management Process, Inor- \\ ganic Soil Fertility Program, Holetta, Ethiopia.
}

\begin{abstract}
How to cite this paper: Egata Shunka, Abebe Chindi, Kassaye Negash, Gebremedhin W/Giorigis, Tesfaye Abebe, Atsede Solomon, Mihiretu Bedasa, Ibrahim Said, Lema Tesema. (2021) Optimum Rate of Blended Fertilizer and Urea Determination for Potato Production under Irrigation Condition in Holetta Research Center, West Showa, Ethiopia. International Journal of the Science of Food and Agriculture, 5(1), 129-139.

DOI: 10.26855/ijfsa.2021.03.017
\end{abstract}

Received: January 23, 2021

Accepted: February 22, 2021

Published: March 10, 2021

*Corresponding author: Egata Shunka, Ethiopian Institute of Agricultural Research, Holetta Agricultural Research Center, Crop Research Process, Potato Research Program, Addis Ababa, Ethiopia.

Email: eshunka2007@gmail.com

\begin{abstract}
Potato is an important food and cash crop in Ethiopia, especially in the high and mid-altitude areas. Lack of scientific recommendation of fertilizer rate to popular varieties limited crop yield, especially under irrigation potential. The research was conducted in Holetta Agricultural Research Center, under irrigation during 2019-2020 off-season to find and recommend the NPSB blended fertilizer and urea rates for potato using rates of blended NPSB + urea $(0,150+150,200+$ $150,250+150,300+150,150+250,200+250,250+250,300+250,150+$ 350, $200+350,250+350,300+350) \mathrm{kg} / \mathrm{ha}$ and recommended $195 \mathrm{~kg} / \mathrm{ha}$ DAP $+165 \mathrm{~kg} / \mathrm{ha}$ urea fertilizer and two varieties Belete and Gudenie. The treatment combinations were arranged in randomized complete block design in factorial arrangement with three replications. The results showed that the rates, variety and interactions were found highly significant for total and marketable tuber yield $\mathrm{t} / \mathrm{ha}$, total and marketable tuber number, average tuber number and weight, stem number and height. From these results, it can be concluded that the responses of potato varieties Belete and Gudenie to NPSB+ urea were highly significant on yield and yield components. Even though, it needs location replication with growing season to offer responsible recommendation, it is better to apply 300 $\mathrm{kg} / \mathrm{ha}$ NPSB $+150 \mathrm{~kg} / \mathrm{ha}$ urea to the Belete potato variety and $150 \mathrm{~kg} / \mathrm{ha}$ NPSB + $250 \mathrm{~kg} / \mathrm{ha}$ urea to Gudenie potato variety for high yield and high economic return in Holetta and areas with similar soil type and agro-ecologies under irrigationie.
\end{abstract}

\section{Keywords}

Recommended Urea and DAP, NPSB, Belete and Gudenie potato varieties

\section{Introduction}

Potato (solanum tuberosu L.) is an important crop in many parts of Ethiopia and it ranks first among root and tuber crops in volume of production and consumed followed by Cassava, Sweet potato and Yam [1]. According to [2], potato is a high potential food security crop in Ethiopia because of its high yielding potential, nutritional quality, short growing period and wider adaptability. Based on area coverage it is second crop in Ethiopia next to Enset (Ensete ventricosum L.) [3]. Potato is an important food and cash crop in central highlands of Ethiopia. The potential attainable average yields of 
the crop on research and farmers' fields are 45 and 25 tons/ha, respectively, while the national average production is limited to about 12.3 tons/ha [4, 1]. Potato is an important food and cash crop in Ethiopia, especially in the high and mid altitude areas. It plays good role in improving the quality of the basic diet in both rural and urban areas of the country [5].

Potato cropping systems help to improve resilience especially among smallholder farmers by providing direct access to nutritious food, increasing household incomes and reducing their liability to food price instability [6]. In Ethiopia, its 'meher' season production area has reached about 66, 926 ha, total production of 921,403.2 ton cultivated by over 1.2 million households [1]. On the other hand, the productivity of this crop in the country is very low $\left(13.8 \mathrm{tha}^{-1}\right)$ compared to the world's average yield of $19 \mathrm{t} \mathrm{ha}^{-1}$ [1]. Utilization of different cropping seasons with in a year especially, irrigation and belg production season was one problem among production declining factors for potato crop. In addition, supporting these two diseases free (late blight) excusing production seasons have double importance for farmers by increasing yield and decreasing cost spent for chemicals to control late blight diseases. Less attention was given from the government and research centers to develop appropriate technology that best go with such growing conditions accompanied by water shortages. These conditions require both short maturing crops and drought tolerant varieties.

Optimal crop production and good quality product is a result of balanced fertilization. In balanced fertilization, soil nutrient content and crop requirement should meet each other. The amount of nutrient depleted per year should be balanced with the amount of fertilizers applied in the year. Use of balanced fertilizers in deficient soils can improve fertilizer-use efficiency and crop profitability. Fertilizer determination research works carried earlier have been limited to the two common macro nutrients ( $\mathrm{N}$ and $\mathrm{P}$ ) and hence the effects of blended fertilizers have not been studied. The ratio of NPSB in blended fertilizer is N: 18.9 P: 37.7, S: 6.95, B: 0.1 [7].

New fertilizer materials with value addition and fortification with secondary and micro nutrients would be required to ensure balanced fertilizer use involving most of the nutrients required by crops [8]. Optimum management of water resources at the farm level is needed in view of increasing water demands, limited resources, and aquifer contamination [9]. When irrigation is required there are many available methods and management strategies. The selection of the method and approach depends on factors such as water availability, crop type, soil characteristics, land topography and associated cost [10]. Furrow irrigation is one of the most frequently used surface irrigation methods in the world [11]. This irrigation method is used mainly to irrigate row crops and orchards. Nowadays, furrow irrigation's importance was gaining attention because of the high cost of energy in pressurized irrigation methods and the incorporation of automation in its operation [10].

The formulation blending fertilizers based on actual need is determined by the combination of crop requirement and soil test level. Lack of appropriate fertilizer blends micro nutrients in fertilizer blends is a national problem constituting a major constraint to crop productivity. The recent completed research and soil tests through the Ethiopian Soil Information System Project, revealed that Ethiopian soils are deficient in various other nutrients that are not provided by DAP and urea [12]. This research was conducted because of lack of research recommendation to the newly formulated fertilizers NPSB $\left(18.9 \% \mathrm{~N}, 37.7 \% \mathrm{P}_{2} \mathrm{O}_{5}, 6.95 \% \mathrm{~S}\right.$, and $\left.0.1 \% \mathrm{~B}\right)$ to overcome soil nutrient deficiencies. Scientific recommendation of fertilizer rate to popular varieties was limited especially under irrigation potential. Poor soil fertility and lack of appropriate fertilizer blends recommendations of micronutrients are the major constrains to crop production. Therefore, this research was conducted to identify and recommend the optimum rate of NPSB blended fertilizer + urea for potato cultivars under irrigation in Holetta Research Center.

\section{Materials and Methods}

The research was conducted in Holetta Agricultural Research Center during 2019-2020 off-seasons comprising two factors; improved potato varieties (Gudenie, Belete) and fertilizer treatments NPSB + Urea fertilizer [0, 300 + 150, 200 $+250,300+350,200+150,250+250$, recommended DAP (195) and Urea (165), $300+250,150+350,150+150$, $250+150$, control, $200+350,250+350,150+250] \mathrm{kg} / \mathrm{ha}$. The blended NPSB contains $18.9 \% \mathrm{~N}, 37.7 \% \mathrm{P}_{2} \mathrm{O}_{5}, 6.95 \%$ $\mathrm{S}$ and $0.1 \% \mathrm{~B}$ while DAP contains $46 \% \mathrm{P}$ and $18 \% \mathrm{~N}$. But, urea contains $46 \% \mathrm{~N}$. The treatment combinations were arranged in randomized complete block design in three replications. Each plot had 4.5 meter width and $3 \mathrm{~m}$ length which forms a block holding 28 treatment or plot. The distance between block was 1.5 meter and between consecutive plots was 1 meter.

\subsection{Experimental procedures}

The land was prepared well by tractor plowing three times until fine tilth was achieved in similar ways of land preparation rule for potato fields in Holetta research center. Sprouted medium-sized seed tubers were planted according to the specified treatments in $75 \mathrm{~cm}$ distance between rows and $30 \mathrm{~cm}$ between plants. Cultivation, weeding and harvesting was done at the appropriate time according to the research recommendations. The soil analyses were done before planting, taking soil samples from a depth of $20-30 \mathrm{~cm}$.

\subsection{Fertilizer application, Harvesting and other cultural practice}

Application of blended NPSB and half urea fertilizers at specified rates was done by banding the granules of fertilizers at depth of 5-10 $\mathrm{cm}$ and $5 \mathrm{~cm}$ away from the seed tuber at planting time while half remaining urea applied at 45 days 
after planting. The experimental field was irrigated before and after planting. After planting, irrigation was done every seven days to field capacity in all the experimental plots by furrow irrigation method. Ten days before harvesting, the haulms of the potato plants were removed using a sickle. Tuber harvesting were done once at proper physiological maturity attainment (70\% leaves withering) as described by [13].

\subsection{Data collections}

Number of main stem /hill, plant height (cm), average tuber number/hill, average tuber weight/ hill, total and marketable tuber number per plot, total and marketable tuber yield t/ha were collected.

\subsection{Statistical Data Analysis}

Data were subjected to analysis of variance (ANOVA) for Randomized Complete Block Design in factorial arrangement by using GLM (general linear model) procedure of SAS 9.2 software [14]. All significant pairs of treatment were compared with the Least Significant Difference (LSD) at 5\% significance level and the partial budget analysis was conducted for the fertilizer rates.

\subsection{Economic Analysis}

Simple partial budget analysis was employed for economic analysis of fertilizer application and it was carried out for average marketable tuber yield data. The possible response of the crop towards the added fertilizer and price of fertilizer during planting finally determines the economic feasibility of fertilizer application [15].

\section{Result}

\subsection{Soil Physico-Chemical properties of Experimental Site}

The result of laboratory analysis selected physico-chemical properties of the soil of excremental site was presented in Table 1 . The $\mathrm{pH}$ of the soil before planting was 5.08. The cation exchange capacity (CEC) of the experimental site was 21.54 meq/100 g soils. The analysis of available P, total N, S and B indicated the experimental soil had value of 8.641 $\mathrm{ppm}, 0.11 \%, 3.9 \%$ and $0.36 \mathrm{mg} / \mathrm{kg}$, respectively. Based on soil analysis result, the experimental site had clay 53.5\%, silt $36.5 \%$ and sand $10 \%$ and categorized as clay loam textural classes.

Table 1. Soil Physical properties of the experimental site before planting

\begin{tabular}{cccc}
\hline Result /Parameters tested & Value & Physical properties & Values \\
\hline pH & 5.08. & \% Clay & 53.5 \\
CEC (meq/100g ) & 21.54 & \% Silt & 36.5 \\
P ppm & 8.641 & \% Sand & 10 \\
N (\%) & 0.11 & Texture class & Clay loam \\
S (\%) & 3.9 & & \\
Av. B (mg/kg) & 0.36 & & \\
\hline
\end{tabular}

\subsection{Number of main Stems Per hill}

The analysis of variance showed that the growing year, fertilizer rates, variety and interaction of fertilizer rates and variety highly significantly affected $(\mathrm{P}<0.001)$ the number of stem number (Table 2 and Table 3$)$. The highest $(4.55$ stem number was recorded from 300 NPSB +150 urea the fertilizer treatment while the lowest (3.18) stem number was recorded from 300 NPSB +250 urea $\mathrm{kg} / \mathrm{ha}$. The recommended DAP and Urea provided SN (4.03) which was significantly different from the highest SN scored from (300 NPSB + 150 urea). The highest (3.98) stem number/hill was recorded from Gudenie variety while the lowest (3.07) stem number was recorded from Belete. Significantly higher stem number (4.7) per hill was produced in 2019 than 2020 (2.35). In the interaction effect of NPSB + urea and varieties, this highest (4.05) stem number was recorded from Gudenie variety, the highest (5.05) stem number was produced at 300 NPSB+150 urea fertilizer treatment, while the lowest (3.43) was recorded from 300 NPSB + 250 urea.

\subsection{Plant Height}

The analysis of variance showed that the NPSB + urea fertilizer rates, variety and growing year as well as the interactions of fertilizer rate and variety were highly significant in affecting $(\mathrm{P}<0.001)$ the plant height (Table 2 and Table 3$)$. The highest plant height $(87.75 \mathrm{~cm})$ was recorded in $200 \mathrm{NPSB}+250$ urea $\mathrm{kg} / \mathrm{ha}$ followed by $86.52 \mathrm{~cm}$ and $85.5 \mathrm{~cm}$ at $300 \mathrm{NPSB}+350$ urea $\mathrm{kg} / \mathrm{ha}$ and $200 \mathrm{NPB}+350$ urea $\mathrm{kg} / \mathrm{ha}$, respectively while the lowest plant height $(64.26 \mathrm{~cm})$ was recorded from control treatment. The recommended DAP and urea's plant height was significantly lower than the highest result mentioned above. The taller plant height was produced by Gudanie and 2020 production year. In the interaction, the highest plant height $(90.43 \mathrm{~cm})$ was recorded from variety Belete at $300 \mathrm{NPSB}+150 \mathrm{urea} \mathrm{kg} / \mathrm{ha}$ fertilizer treatment while the lowest plant height $(65.50 \mathrm{~cm})$ produced from control. The recommended DAP and urea provided 
significantly lower plant height than the highest plant height mentioned above. Variety Gudenie produced the highest $(87.81 \mathrm{~cm})$ plant height at 300 NPSB +350 urea fertilizer treatments while the lowest $(63.03 \mathrm{~cm})$ one was recorded from control. Recommended DAP and urea gave significantly lower plant height $(73.38 \mathrm{~cm})$ with Gudenie variety than the highest plant height mentioned for Gudenie.

Table 2. Effect of fertilizer rates, variety and growing season on stem number, plant height, yield and yield component

\begin{tabular}{|c|c|c|c|c|c|c|c|c|}
\hline Fertilizer NPSB+Urea kg/ha & $\mathrm{SN}$ & $\mathrm{PH}$ & ATN & ATW & TTN & MTY t/ha & TTY t/ha & MTN \\
\hline 0 & 3.37cdefg & $64.26 \mathrm{~h}$ & $6.62 \mathrm{~g}$ & $0.61 \mathrm{~g}$ & $154.83 \mathrm{~h}$ & $22.96 \mathrm{~h}$ & $28.25 h$ & $100.42 \mathrm{~g}$ \\
\hline $300+150$ & $4.55 a$ & 77.75defg & $9.54 \mathrm{bc}$ & $0.92 \mathrm{bc}$ & $271.92 \mathrm{a}$ & $36.36 b$ & $42.27 b$ & $180.54 a$ \\
\hline $200+250$ & $3.64 c$ & $87.75 a$ & $8.10 \mathrm{f}$ & $0.77 \mathrm{f}$ & 233.83efg & $29.85 g$ & $34.01 \mathrm{~g}$ & $157.83 \mathrm{ef}$ \\
\hline $300+350$ & 3.36cdefg & $86.52 a$ & $8.77 d$ & $0.82 \mathrm{e}$ & 242.92de & 32.95ef & 37.07ef & 175.50abc \\
\hline $200+150$ & $3.63 c$ & 78.60def & $9.39 b c$ & $0.91 \mathrm{bc}$ & 240.08def & $35.74 b c$ & $39.27 d$ & $168.00 \mathrm{~cd}$ \\
\hline $250+250$ & 3.49cde & $83.03 c$ & $9.12 \mathrm{~cd}$ & $0.84 \mathrm{e}$ & $262.58 \mathrm{ab}$ & 33.74de & $37.38 \mathrm{e}$ & $178.50 \mathrm{ab}$ \\
\hline 195 DAP+165urea & $4.03 \mathrm{~b}$ & $75.53 g$ & $8.21 \mathrm{f}$ & $0.85 \mathrm{de}$ & 240.42def & 33.06ef & $37.43 e$ & $171.75 b c$ \\
\hline $300+250$ & $3.09 \mathrm{~g}$ & 83.71bc & $9.61 b$ & $0.91 b c$ & $269.00 \mathrm{a}$ & 34.92cd & $40.60 c$ & $151.67 f$ \\
\hline $150+350$ & 3.18efg & 76.31fg & 8.23ef & $0.85 \mathrm{e}$ & $225.92 \mathrm{~g}$ & 33.84de & $35.88 f$ & 161.17de \\
\hline $150+150$ & $3.46 \mathrm{cdef}$ & 79.02de & 8.69de & $0.82 \mathrm{e}$ & 239.58def & $31.74 \mathrm{f}$ & 36.47ef & 155.67ef \\
\hline $250+150$ & $3.67 c$ & 76.80efg & $10.27 a$ & $0.93 b$ & $256.25 b c$ & 34.73cd & $41.94 \mathrm{~b}$ & 162.25de \\
\hline $200+350$ & 3.23defg & $80.06 \mathrm{~d}$ & $9.15 b c d$ & $0.89 \mathrm{bcd}$ & 246.83cd & $34.54 \mathrm{~cd}$ & 39.49cd & 158.08ef \\
\hline $250+350$ & $3.54 \mathrm{~cd}$ & $85.50 \mathrm{ab}$ & 8.73d & $0.89 \mathrm{bcd}$ & 232.00fg & $34.75 \mathrm{~cd}$ & $39.24 d$ & 159.42ef \\
\hline $150+250$ & $3.14 f g$ & 77.44efg & 8.81d & $0.98 a$ & 232.83efg & $38.44 a$ & 43.63a & 176.17abc \\
\hline LSD & 0.3486 & 2.4252 & 0.4732 & 0.0385 & 10.147 & 1.3552 & 1.2935 & 8.4976 \\
\hline P-VALUE & $<0.0001$ & $<0.0001$ & $<0.0001$ & $<0.0001$ & $<0.0001$ & $<0.0001$ & $<0.0001$ & $<0.0001$ \\
\hline \multicolumn{9}{|c|}{ Variety } \\
\hline Belete & $3.07 \mathrm{~b}$ & 81.92a & $9.00 \mathrm{a}$ & $1.00 \mathrm{a}$ & 237.30b & $38.79 a$ & $44.38 \mathrm{a}$ & 160.71 \\
\hline Gudenie & $3.98 a$ & $76.98 b$ & $8.61 b$ & $0.72 b$ & 241.13a & $28.00 \mathrm{~b}$ & $31.75 b$ & 161.71 \\
\hline LSD & 0.1318 & 0.9166 & 0.1789 & 0.0146 & & 0.5122 & 0.4889 & \\
\hline P-value & $<0.0001$ & $<0.0001$ & $<0.0001$ & $<0.0001$ & NS & $<0.0001$ & $<0.0001$ & NS \\
\hline \multicolumn{9}{|c|}{ Growing year } \\
\hline 2019 & $4.70 \mathrm{a}$ & $60.14 \mathrm{~b}$ & 8.91a & $0.70 \mathrm{~b}$ & 205.35b & $24.48 b$ & $31.47 \mathrm{~b}$ & $105.02 b$ \\
\hline 2020 & $2.35 b$ & $98.76 a$ & $8.70 \mathrm{~b}$ & $1.02 \mathrm{a}$ & 273.08a & $42.32 \mathrm{a}$ & $44.67 a$ & 217.41a \\
\hline LSD & 0.1318 & 0.9166 & 0.1789 & 0.0146 & 3.8351 & 0.5122 & 0.4889 & 3.2118 \\
\hline P-value & $<0.0001$ & $<0.0001$ & 0.0271 & $<0.0001$ & $<0.0001$ & $<0.0001$ & $<0.0001$ & $<0.0001$ \\
\hline $\mathrm{CV}$ & 12.22 & 3.77 & 6.643 & 5.55 & 5.24 & 5.01 & 4.00 & 6.52 \\
\hline
\end{tabular}

$\mathrm{SN}=$ stem number, $\mathrm{PH}=$ plant height in $\mathrm{cm}$, ATN = Average tuber number per plant, ATW = Average tuber number per hill in kg, TTN = Total tuber number per plot, MTN = Marketable tuber number per plot, TTY t/ha = Total tuber yield ton per hectare, and MTY $\mathrm{t} / \mathrm{ha}=$ marketable tuber number ton per hectare.

\subsection{Average Tuber Number (ATN)}

The analysis of variance showed that effect of blended fertilizer NPSB, varieties and the interaction of variety and fertilizer rates were highly significant in affecting $(\mathrm{P}<0.001)$ average tuber number (Table 2 and Table 3 ) while the growing year effect was significant. The highest average tuber (ATN) number (10.27) and the lowest ATN (6.62) was recorded at fertilizer rates of $250 \mathrm{NPSB}+150$ urea $\mathrm{kg} / \mathrm{ha}$ and control consecutively. The recommended urea and DAP provided ATN (8.21) which was significantly highest ATN from control and lower from the highest average tuber number. The highest (9.00) average tuber number (ATN) was recorded from Belete variety while the lowest (8.61) average tuber number (ATN) was recorded from variety Gudenie not received fertilizers. In their interaction, the highest (10.36/hill) average tuber number was recorded from variety Belete at 200 NPSB +150 urea $\mathrm{kg} / \mathrm{ha}$ treatment and the lowest 
(7.00/hill) average tuber number produced from Belete variety grown without fertilizers while Gudenie provided the highest $10.83 \mathrm{ATN} / \mathrm{hill}$ at $300 \mathrm{NPSB}+150$ urea $\mathrm{kg} / \mathrm{ha}$ and lowest $6.24 \mathrm{ATN} / \mathrm{hill}$ at control treatment.

\subsection{Average Tuber Weight (ATW)}

Average tuber weight was highly significantly affected by blended fertilizer NPSB, variety, growing year and the interaction of NPSB and variety $(\mathrm{p}<0.001)$ (Table 2 and Table 3). The highest ATW $0.98 \mathrm{~kg} / \mathrm{hill}$ observed on fertilizer rate $150 \mathrm{NPSB}+250$ urea $\mathrm{kg} / \mathrm{ha}$ and the lowest ATW $0.61 \mathrm{~kg} / \mathrm{hill}$ was seen in control. The recommended urea and DAP provided $0.85 \mathrm{~kg} /$ hill ATW which was significantly different from the highest average tuber weight scored from150 NPSB + 250 urea $\mathrm{kg} / \mathrm{ha}$. The highest $1.00 \mathrm{~kg} / \mathrm{hill}$ average tuber weight was recorded from variety Belete while lowest $0.72 \mathrm{~kg} / \mathrm{hill}$ average tuber weight was recorded from variety Gudenie. In the interaction, the highest $1.14 \mathrm{~kg} / \mathrm{hill}$ average tuber weight was recorded from variety Belete at 250 NPSB +150 urea $\mathrm{kg} / \mathrm{ha}$ fertilizer treatment followed by $1.08 \mathrm{~kg} / \mathrm{hill}$ at $300 \mathrm{NPSB} \mathrm{kg} / \mathrm{ha}+150 \mathrm{~kg} / \mathrm{ha}$ urea and $300 \mathrm{NPSB} \mathrm{kg} / \mathrm{ha}$ and $250 \mathrm{~kg} / \mathrm{ha}$ urea while the lowest $0.70 \mathrm{~kg} / \mathrm{hill}$ average tuber weight obtained from control treatment. In line with this Gudenie provided its own highest $0.84 \mathrm{~kg} / \mathrm{hill}$ ATW at $150 \mathrm{NPSB} \mathrm{kg} / \mathrm{ha}+250 \mathrm{~kg} / \mathrm{ha}$ urea and $300 \mathrm{~kg} / \mathrm{ha}$ NPSB$+150 \mathrm{~kg} / \mathrm{ha}$ urea while the lowest for Gudenie was produced at control treatment.

\subsection{Total Tuber Number (TTN)}

The results of the analysis of variance showed that the effect of blended fertilizer NPSB, growing year, and the interaction of rates and variety were highly significant $(\mathrm{P}<0.001)$ (Table 2 and Table 3 ) while the variety was not significant. The highest TTN (271.92) was recorded at 300 NPSB + 150urea kg/ha followed by 269 TTN at $300 \mathrm{~kg} / \mathrm{ha}$ NPSB +250 $\mathrm{kg} / \mathrm{ha}$ urea and $262.58 \mathrm{TTN}$ at $250 \mathrm{~kg} / \mathrm{ha}$ NPSB $+250 \mathrm{~kg} / \mathrm{ha}$ urea while the lowest $154.58 \mathrm{TTN}$ was recorded in control. The recommended DAP and urea provided 240.42 TTN which was significantly different from the highest TTN scored from 300 NPSB + 150 urea kg/ha. The application of blended fertilizer 300 NPSB kg/ha $+150 \mathrm{~kg} / \mathrm{ha}$ urea increased total tuber number from 154.58 to 271.92 which could be calculated to 76 \% increase. In 2020, the higher tuber number 273.08 was produced while in 2019 lower TTN 205.35 was harvested.

In interaction cases, the highest 270.17 total tuber number was recorded from Belete variety at $150 \mathrm{~kg} / \mathrm{ha} \mathrm{NPSB}+$ $150 \mathrm{~kg} / \mathrm{ha}$ urea followed by 167.50 TTN produced at $300 \mathrm{~kg} / \mathrm{ha}$ NPSB $+150 \mathrm{~kg} / \mathrm{ha}$ urea while Belete variety's lowest TTN 149.33 was produced from plots not treated with fertilizers. Gudenie provided its highest $284.50 \mathrm{TTN}$ at $250 \mathrm{~kg} / \mathrm{ha}$ NPSB + $150 \mathrm{~kg} / \mathrm{ha}$ urea followed by 276.33 at $300 \mathrm{~kg} / \mathrm{ha}$ NPSB + $150 \mathrm{~kg} / \mathrm{ha}$ urea and $300 \mathrm{~kg} / \mathrm{ha} \mathrm{NPSB}+250 \mathrm{~kg} / \mathrm{ha}$ urea while lowest 160.33 total tuber number was recorded from control. Gudenie's highest 284.50 TTN was higher than Belete's highest TTN (267.17).

\subsection{Marketable Tuber Number (MTN)}

Based on the analysis of variance, the effect of blended fertilizer NPSB, growing year and the interaction of fertilizer rates and variety were highly significant $(\mathrm{P}<0.001)$ (Table 2 and Table 3 ). The highest 180.54 marketable tuber number was recorded from $300 \mathrm{~kg} / \mathrm{ha}$ NPSB $+150 \mathrm{~kg} /$ ha urea treatment and par with the treatments that received $250 \mathrm{~kg} / \mathrm{ha}$ NPSB + $250 \mathrm{~kg} / \mathrm{ha}$ urea (178.50/plot), $300 \mathrm{~kg} / \mathrm{ha}$ NPSB + $350 \mathrm{~kg} / \mathrm{ha}$ urea (176.17/plot) and $300 \mathrm{~kg} / \mathrm{ha}$ NPSB + 350 $\mathrm{kg} / \mathrm{ha}$ urea while the lowest $100.42 \mathrm{MTN}$ was recorded from not fertilized plot. Applying $300 \mathrm{~kg} / \mathrm{ha}$ NPSB $+150 \mathrm{~kg} / \mathrm{ha}$ urea fertilizer increased MTN from 100.42 to 180.54 by $84 \%$. The recommended urea and DAP provided 171.75 MTN which was significantly lower than the highest MTN but, in par with MTN recorded at $250 \mathrm{~kg} / \mathrm{ha} \mathrm{NPSB}+250 \mathrm{~kg} / \mathrm{ha}$

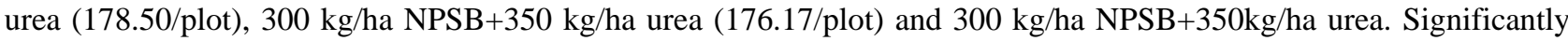
higher 217.41 MTN was produced in 2020 while lower 105.02 MTN harvested from 2019 production season. The highest 190.83/plot marketable tuber number was recorded from Belete variety at $150 \mathrm{~kg} / \mathrm{ha} \mathrm{NPSB}+250 \mathrm{~kg} / \mathrm{ha}$ urea followed by 187.00 which harvested from $150 \mathrm{~kg} / \mathrm{ha}$ NPSB+ $150 \mathrm{~kg} /$ ha urea while the lowest 88.33/plot marketable tuber number was recorded from control. Gudenie gave its highest 208.00 MTN at recommended urea and DAP followed by $200.17 \mathrm{MTN}$ at $300 \mathrm{~kg} / \mathrm{ha}$ NPSB+ $350 \mathrm{~kg} /$ ha urea while its lowest $112.50 \mathrm{MTN}$ was harvested from control treatment. Application of $150 \mathrm{~kg} / \mathrm{ha}$ NPSB $+250 \mathrm{~kg} / \mathrm{ha}$ urea to Belete Gave an advantage of increasing MTN from 88.33 to 190.83 which was $116 \%$.

\subsection{Total Tuber Yield (TTY t/ha)}

The analysis of variance showed that the response of blended NPSB fertilizer, varieties, growing year, and the interactions of variety and rates were highly significantly affected $(\mathrm{P}<0.001)$ total tuber yield (Table 2 and Table 3). The maximum total tuber yield of $43.63 \mathrm{t} / \mathrm{ha}$ was produced on the application of $150 \mathrm{NPSB}+250$ urea $\mathrm{kg} / \mathrm{ha}$ while the lowest $28.25 \mathrm{t} / \mathrm{ha}$ total tuber yield (TTY) was recorded from control. It showed a $54.44 \%$ difference from control. Belete gave significantly higher 44.38 t/ha TTY while Gudenie produced 31.75 t/ha. Significantly higher 44.67 t/ha TTY was harvested in 2020 than in 2019(31.47 t/ha). 
In the interaction of variety and rates, Belete gave its maximum 51.16 t/ha tuber yield at $300 \mathrm{~kg} / \mathrm{ha}$ NPSB +150 $\mathrm{kg} / \mathrm{ha}$ urea followed by $49.92 \mathrm{t} / \mathrm{ha}$ at $150 \mathrm{~kg} / \mathrm{ha} \mathrm{NSB}+250 \mathrm{~kg} / \mathrm{ha}$ urea while the lowest $33.26 \mathrm{t} / \mathrm{ha}$ tuber yield was recorded from control. Application of $250 \mathrm{NPSB}+150$ urea kg/ ha to Gudenie triggered to produce maximum yield 39.09 $\mathrm{kg} / \mathrm{ha}$ followed by $150 \mathrm{~kg} / \mathrm{ha}$ NPSB $+250 \mathrm{~kg} / \mathrm{ha}$ urea while the lowest yield $23.25 \mathrm{t} / \mathrm{ha}$ was obtained from control. Belete variety showed tuber yield increment from 33.26 to 51.16 t/ha as a result of increasing fertilizers from 0-300NPSB +150 urea $\mathrm{kg} / \mathrm{ha}$ which is $54 \%$ while Gudenie provided the advantage of $68.13 \%$ tuber yield increase due to increasing fertilizers from 0 to150 kg/ha NPSB + $250 \mathrm{~kg} / \mathrm{ha}$ urea.

Table 3. The interaction effect of fertilizer rates and variety on plant height, yield and yield component of Belete and Gudenie variety

\begin{tabular}{|c|c|c|c|c|c|c|c|c|c|}
\hline Variety & $\begin{array}{l}\text { Fertilizer NPSB+Urea } \\
\text { kg/ha }\end{array}$ & SN & PH(cm) & $\begin{array}{l}\text { ATN/ } \\
\text { plant }\end{array}$ & $\begin{array}{c}\text { ATW } \\
\text { (kg/ plant) }\end{array}$ & $\begin{array}{l}\text { TTN/ } \\
\text { plot }\end{array}$ & $\begin{array}{l}\text { MTN/ } \\
\text { plot }\end{array}$ & $\begin{array}{l}\text { TTY } \\
\text { t/ha }\end{array}$ & $\begin{array}{c}\text { MTY } \\
\text { t/ha }\end{array}$ \\
\hline \multirow{12}{*}{ Belete } & 0 & 2.48 & 65.50 & 7.00 & 0.70 & 149.33 & 88.33 & 33.26 & 25.81 \\
\hline & $300+150$ & 4.05 & 79.99 & 9.27 & 1.08 & 267.50 & 176.33 & 51.16 & 44.33 \\
\hline & $200+250$ & 3.63 & 88.03 & 8.38 & 0.84 & 239.83 & 167.83 & 37.34 & 33.36 \\
\hline & $300+350$ & 2.65 & 85.23 & 7.88 & 0.94 & 214.17 & 150.83 & 42.94 & 37.21 \\
\hline & $200+150$ & 3.15 & 82.83 & 10.36 & 1.14 & 242.83 & 172.00 & 47.46 & 43.36 \\
\hline & $250+250$ & 3.05 & 80.68 & 9.03 & 1.04 & 252.50 & 184.33 & 46.23 & 40.83 \\
\hline & 195DAP +165urea & 3.58 & 77.68 & 7.93 & 0.93 & 224.50 & 135.50 & 40.31 & 34.84 \\
\hline & $300+250$ & 2.75 & 90.43 & 9.84 & 1.08 & 261.67 & 177.67 & 47.82 & 40.10 \\
\hline & $150+350$ & 2.91 & 74.71 & 8.79 & 0.93 & 245.33 & 163.83 & 41.53 & 39.86 \\
\hline & $150+150$ & 2.63 & 84.21 & 9.56 & 1.03 & 270.17 & 187.00 & 45.51 & 40.90 \\
\hline & $250+150$ & 3.03 & 82.13 & 9.71 & 1.01 & 228.00 & 141.33 & 44.79 & 35.71 \\
\hline & $200+350$ & 2.87 & 86.01 & 9.27 & 1.05 & 222.17 & 142.33 & 46.39 & 40.49 \\
\hline \multirow{18}{*}{ Gudenie } & $250+350$ & 3.40 & 87.79 & 9.37 & 1.05 & 241.33 & 171.83 & 46.75 & 41.58 \\
\hline & $150+250$ & 2.83 & 81.71 & 9.57 & 1.12 & 262.83 & 190.83 & 49.92 & 44.76 \\
\hline & 0 & 4.26 & 63.03 & 6.24 & 0.53 & 160.33 & 112.50 & 23.25 & 20.11 \\
\hline & $300+150$ & 5.05 & 75.52 & 9.81 & 0.77 & 276.33 & 184.75 & 33.39 & 28.40 \\
\hline & $200+250$ & 3.65 & 87.48 & 7.82 & 0.71 & 227.83 & 147.83 & 30.69 & 26.35 \\
\hline & $300+350$ & 4.08 & 87.81 & 9.66 & 0.70 & 271.67 & 200.17 & 31.20 & 28.68 \\
\hline & $200+150$ & 4.12 & 74.38 & 8.42 & 0.68 & 237.33 & 164.00 & 31.08 & 28.12 \\
\hline & $250+250$ & 3.93 & 85.38 & 9.22 & 0.64 & 272.67 & 172.67 & 28.54 & 26.65 \\
\hline & 195 DAP +165urea & 4.48 & 73.38 & 8.49 & 0.78 & 256.33 & 208.00 & 34.56 & 31.27 \\
\hline & $300+250$ & 3.43 & 77.00 & 9.39 & 0.75 & 276.33 & 125.67 & 33.39 & 29.74 \\
\hline & $150+350$ & 3.45 & 77.91 & 7.68 & 0.76 & 206.50 & 158.50 & 30.24 & 27.83 \\
\hline & $150+150$ & 4.29 & 73.83 & 7.82 & 0.62 & 209.00 & 124.33 & 27.43 & 22.59 \\
\hline & $250+150$ & 4.31 & 71.47 & 10.83 & 0.84 & 284.50 & 183.17 & 39.09 & 33.75 \\
\hline & $200+350$ & 3.60 & 74.12 & 9.02 & 0.73 & 271.50 & 173.83 & 32.59 & 28.59 \\
\hline & $250+350$ & 3.68 & 83.20 & 8.10 & 0.72 & 222.67 & 147.00 & 31.73 & 27.92 \\
\hline & $150+250$ & 3.45 & 73.18 & 8.06 & 0.84 & 202.83 & 161.50 & 37.34 & 32.12 \\
\hline & $\mathrm{CV}$ & 12.22 & 3.77 & 3.77 & 5.55 & 5.24 & 6.52 & 6.52 & 5.01 \\
\hline & P-value & $<0.0001$ & $<0.0001$ & $<0.0001$ & $<0.0001$ & $<0.0001$ & $<0.0001$ & $<0.0001$ & $<0.0001$ \\
\hline
\end{tabular}

$\mathrm{SN}=$ stem number, $\mathrm{PH}=$ plant height in $\mathrm{cm}, \mathrm{ATN}=$ Average tuber number per plant, ATW = Average tuber number per hill in $\mathrm{kg}$, TTN = Total tuber number per plot, MTN = Marketable tuber number per plot, TTY t/ha = Total tuber yield ton per hectare, and MTY $\mathrm{t} / \mathrm{ha}=$ marketable tuber number ton per hectare. 


\subsection{Marketable Tuber Yield (MTY)}

Marketable tuber yield was highly significantly affected by the blended NPSB fertilizer, varieties, growing year, and the interactions of variety and rates (Table 2 and Table 3). The highest $38.44 \mathrm{t} /$ ha marketable tuber yield (MTY) was recorded from $150 \mathrm{NPSB}+250$ urea kg/ha while the lowest $22.96 \mathrm{t} / \mathrm{ha}$ MTY was harvested from control. Belete variety provided significantly higher 38.79 t/ha MTY while Gudenie produced 28.00 t/ha lower MTY. In 2020, significantly higher 42.32 t/ha MTY was harvested while lower 24.48 t/ha MTY was recorded in the 2019 cropping season.

The blended fertilizer 150 NPSB+250 Urea kg/ ha applied on Belete resulted in the production of Belete's maximum 44.76 t/ha MTY followed by application of $300 \mathrm{~kg} / \mathrm{ha}$ NPSB + $150 \mathrm{~kg} / \mathrm{ha}$ urea, 44.33 t/ha MTY. 33.75 t/ha MTY was the maximum MTY of Gudenie harvested at the application of $250 \mathrm{~kg} / \mathrm{ha}$ NPSB $+150 \mathrm{~kg} / \mathrm{ha}$ urea. Plot not received fertilizers gave the lowest MTY of 25.81 t/ha and 20.11 t/ha with Belete and Gudenie varieties respectively. When compared with control Belete variety showed a 73.42\% MTY increase while Gudenie showed 68\%.

\subsection{Partial budget analysis}

Table 4. Partial Budget analysis

\begin{tabular}{|c|c|c|c|c|c|c|c|c|c|c|c|c|}
\hline \multirow[b]{2}{*}{ NPSB } & \multirow[b]{2}{*}{ Urea } & \multicolumn{11}{|c|}{ Belete } \\
\hline & & $\begin{array}{c}\text { Variable } \\
\text { Costs ETB }\end{array}$ & \multicolumn{2}{|c|}{$\begin{array}{l}\text { Maximum } \\
\text { Cost ETB }\end{array}$} & $\begin{array}{l}\text { Grows } \\
\text { yield } \\
\text { t/ha }\end{array}$ & $\begin{array}{l}\text { Adjusted } \\
\text { yield t/ha }\end{array}$ & \multicolumn{2}{|c|}{$\begin{array}{c}\text { Grows } \\
\text { Benefit } \\
\text { ETB }\end{array}$} & \multicolumn{2}{|c|}{$\begin{array}{l}\text { Net benefit } \\
\text { ETB }\end{array}$} & $\begin{array}{c}\text { Maximum } \\
\text { Benefit } \\
\text { ETB }\end{array}$ & $\begin{array}{c}\text { Maximum } \\
\text { Rate of } \\
\text { Return\% }\end{array}$ \\
\hline 0 & 0 & 0 & & & 25.81 & 23.23 & \multicolumn{2}{|c|}{$232,290.00$} & \multicolumn{2}{|c|}{$232,290.00$} & & \\
\hline 150 & 150 & 4,050 & \multicolumn{2}{|c|}{4,050} & 40.9 & 36.81 & \multicolumn{2}{|c|}{$368,100.00$} & \multicolumn{2}{|c|}{$364,050.00$} & $\underset{n}{131,760.0}$ & 3,253.33 \\
\hline 200 & 150 & 4,750 & \multicolumn{2}{|c|}{700} & 43.36 & 39.02 & \multicolumn{2}{|c|}{$390,240.00$} & \multicolumn{2}{|c|}{$385,490.00$} & $21,440.00$ & 3,062.86 \\
\hline 150 & 250 & 5,350 & \multicolumn{2}{|c|}{600} & 44.76 & 40.28 & \multicolumn{2}{|c|}{$402,840.00$} & \multicolumn{2}{|c|}{$397,490.00$} & $12,000.00$ & $2,000.00$ \\
\hline 250 & 150 & 5,450 & \multicolumn{2}{|c|}{100} & 35.71 & 32.14 & \multicolumn{2}{|c|}{$321,390.00$} & \multicolumn{2}{|c|}{$315,940.00$} & & \\
\hline 243 & 165 & 5,547 & \multicolumn{2}{|c|}{97} & 34.84 & 31.36 & \multicolumn{2}{|c|}{$313,560.00$} & \multicolumn{2}{|c|}{$308,013.00$} & & \\
\hline 200 & 250 & 6,050 & 50 & & 33.36 & 30.02 & 300 & 40.00 & 294 , & 90.00 & & \\
\hline 300 & 150 & 6,150 & 10 & & 44.33 & 39.90 & 398 & 70.00 & 392, & 320.00 & $98,630.00$ & $98,630.00$ \\
\hline 150 & 350 & 6,650 & 50 & & 39.86 & 35.87 & 358 & 40.00 & 352, & 90.00 & & \\
\hline 250 & 250 & 6,750 & 10 & & 40.83 & 36.75 & 367 & 70.00 & 360 & 720.00 & $8,630.00$ & $8,630.00$ \\
\hline 200 & 350 & 7,350 & 60 & & 40.49 & 36.44 & 364 & 10.00 & 357, & 60.00 & & \\
\hline 300 & 250 & 7,450 & 10 & & 40.1 & 36.09 & 360 & 00.00 & 353 & 150.00 & & \\
\hline 250 & 350 & 8,050 & 60 & & 41.58 & 37.42 & 374 & 20.00 & 366 , & 70.00 & $12,720.00$ & $2,120.00$ \\
\hline 300 & 350 & 8,750 & 70 & & 37.21 & 33.49 & 334 & 90.00 & 326 , & 40.00 & & \\
\hline & & & & & & Gude & & & & & & \\
\hline 0 & 0 & 0 & & 20.11 & 18.10 & 180,99 & & 180,9 & 0.00 & & & \\
\hline 150 & 150 & 4,050 & 4,050 & 22.59 & 20.33 & 203,31 & & 199,2 & 0.00 & 18,27 & .00 & 451.11 \\
\hline 200 & 150 & 4,750 & 700 & 28.12 & 25.31 & 253,08 & & 248,3 & 0.00 & 49,07 & .00 & $7,010.00$ \\
\hline 150 & $250 `$ & 5,350 & 600 & 32.12 & 28.91 & 289,08 & & 283,7 & 0.00 & 35,40 & .00 & $5,900.00$ \\
\hline 250 & 150 & 5,450 & 100 & 33.75 & 30.38 & 303,75 & & 298,3 & 0.00 & 14,57 & .00 & $14,570.00$ \\
\hline 243 & 165 & 5,547 & 97 & 31.27 & 28.14 & 281,43 & & 275,8 & 3.00 & & & \\
\hline 200 & 250 & 6,050 & 503 & 26.35 & 23.72 & 237,15 & & 231,1 & 0.00 & & & \\
\hline 300 & 150 & 6,150 & 100 & 28.4 & 25.56 & 255,60 & & 249,4 & 0.00 & 18,35 & .00 & $18,350.00$ \\
\hline 150 & 350 & 6,650 & 500 & 27.83 & 25.05 & 250,47 & & 243,8 & 0.00 & & & \\
\hline 250 & 250 & 6,750 & 100 & 26.65 & 23.99 & 239,85 & & 233,1 & 0.00 & & & \\
\hline 200 & 350 & 7,350 & 600 & 28.59 & 25.73 & 257,31 & & 249,9 & 0.00 & 16,86 & .00 & $2,810.00$ \\
\hline 300 & 250 & 7,450 & 100 & 29.74 & 26.77 & 267,66 & & 260,2 & 0.00 & 10,25 & .00 & $10,250.00$ \\
\hline 250 & 350 & 8,050 & 600 & 27.92 & 25.13 & 251,28 & & 243,2 & 0.00 & & & \\
\hline 300 & 350 & 8,750 & 700 & 28.68 & 25.81 & 258,12 & & 249,3 & 0.00 & 6,140 & & 877.14 \\
\hline
\end{tabular}

Price for $1 \mathrm{~kg}$ potato was $8 \mathrm{ETB}$ and yield adjustment factor $10 \%$. 
The highest net benefit (397,490.00ETB) was gained from $150 \mathrm{~kg} / \mathrm{ha}$ NPSB $+2,500 \mathrm{~kg} / \mathrm{ha}$ urea followed by 300kg/ha on Belete variety (Table 4). The highest 98,630\% maximum rate of return obtained from $300 \mathrm{~kg} / \mathrm{ha} \mathrm{NPSB}+150 \mathrm{~kg} / \mathrm{ha}$ urea while the lowest 2,000\% maximum rate of return was gained from $150 \mathrm{~kg} / \mathrm{ha}$ NPSB $+2,500 \mathrm{~kg} / \mathrm{ha}$ urea among undominated treatments (Table 4). This means, the investment return increased by $98,630.00 \%$ at an application rate of $300 \mathrm{~kg} / \mathrm{ha}$ NPSB $+150 \mathrm{~kg} / \mathrm{ha}$ urea. The highest 298,300.00 ETB net benefit from Gudenie variety was obtained at 250 $\mathrm{kg} / \mathrm{ha}$ NPSB $+150 \mathrm{~kg} / \mathrm{ha}$ urea whiles the lowest 18,0990.00 ETB net benefit gained from the control treatment (Table 4). The highest 18,350.00\% maximum rate of return was gained from $300 \mathrm{~kg} / \mathrm{ha}$ NPSB $+150 \mathrm{~kg} / \mathrm{ha}$ urea while the lowest $451.11 \%$ maximum rate of return was from $150 \mathrm{~kg} / \mathrm{ha}$ NPSB + $150 \mathrm{~kg} / \mathrm{ha}$ urea among un dominated treatment.

\section{Discussions}

The $\mathrm{pH}$ of the soil was 5.08 which is highly acidic according to the rating of [16]. The $\mathrm{pH}$ of the soil between 5.00 and 7.55 is found within a suitable range for crop Production [17]. According to [18] report, the $\mathrm{pH}$ range of most crops was 4-8 because of the variable optimum $\mathrm{pH}$ requirement of different crops. Potato requires the $\mathrm{pH}$ range for production is 4.5-7.5 [19]. Thus, the $\mathrm{pH}$ of the experimental soil is almost within the range for productive soils for the potato crop. The soil of experimental land was containing low level of available P [20] and total nitrogen [16]. Its particle size distribution was in clay loam class as each category was $53.5 \%$ clay, $36.5 \%$ silt, and $10 \%$ sand. The Sulfur and Boron range was very low [16]. The soil was in general, in the low level of available macro nutrient p, total nitrogen, sulfur, and micronutrient boron. The potato grown under irrigation in the soils of Holetta Research Center requires higher addition of these lacking nutrient for the intended yield increase and soil nutrient balance.

Stem number was highly significantly affected by fertilizer rates, variety, and growing year. With growing fertilizer rates the stem number grew and reached a maximum at $300 \mathrm{~kg} / \mathrm{ha}$ NPSB and $150 \mathrm{~kg} / \mathrm{ha}$ urea. In agreement with the result, [21] reported that increasing the rates of blended fertilizers significantly increased the number of stem per hill. This author also indicated variability in stem numbers between varieties. In line with the result, [22] indicated that Belete and Gudenie produced a significantly higher number of main stems per hill (6.52 and 6.89), respectively. Similarly, [23] average main stem number per hill was significantly $(p<0.01)$ influenced by the interaction of the genotype and growing environment. The current study is again confirmed with the report of $[24,25]$ the number of stems per plant (hill) influenced by variety.

Similarly, plant height was also highly significantly affected by fertilizer rated, variety, growing environment, and interaction of rates and variety. In this result, the highest plant height $(87.75 \mathrm{~cm})$ was recorded in $200 \mathrm{NPSB}+250$ urea $\mathrm{kg} / \mathrm{ha}$ which is on par with $86.52 \mathrm{~cm}$ and $85.5 \mathrm{~cm}$ at $300 \mathrm{NPSB}+350 \mathrm{urea} \mathrm{kg} / \mathrm{ha}$ and $200 \mathrm{NPB}+350 \mathrm{urea} \mathrm{kg} / \mathrm{ha}$, respectively while the lowest plant height $(64.26 \mathrm{~cm})$ was recorded from the control treatment. These results were in agreement with the finding of [21] who reported the highest plant height $65.9 \mathrm{~cm}$ at the application of $350 \mathrm{~kg}$ NPSB/ha fertilizer while the lowest plant height $54.85 \mathrm{~cm}$ was recorded from unfertilized treatment. According to the report of [26], the tallest potato plants were observed in response to increasing the rate of NPS application to 272 and $281.75 \mathrm{~kg} / \mathrm{ha}$. Report of [27] also showed that maximum plant height of $92.66 \mathrm{~cm}$ was recorded against the application of $150 \mathrm{~kg}$ N/ha which was statistically higher than the rest of the treatments and the minimum $54.08 \mathrm{~cm}$ was found in the application of $0 \mathrm{~kg} \mathrm{~N} / \mathrm{ha}$. In line with this, the report of [28] also indicated increasing nitrogen 0 to $168 \mathrm{~kg} /$ ha increased the height of potato plants from 34.00 to $88.67 \mathrm{~cm}$. In agreement with the report of [26] the longest plant height was observed on variety Belete supplied with $281.75 \mathrm{~kg} / \mathrm{ha}$ NPS fertilizer. These results were in conformity with the following findings $[23,29,30]$.

The average tuber number and weight were highly significantly affected by rates, variety, and interaction of variety and rates. The highest average tuber number and weight were recorded at $250 \mathrm{~kg} / \mathrm{ha}$ NPSB+150 kg/ha urea and $150 \mathrm{~kg}$ NPSB $+250 \mathrm{~kg} / \mathrm{ha}$ urea respectively while the lowest was harvested from an unfertilized plot. These results were in agreement with [27] finding that mentioned increasing the rate of nitrogen increase total tuber yield/plot, average tuber weight, marketable tuber weight, unmarketable tuber weight, and total tuber number/ plant. The present study is in line with the finding of [31] increasing the application of $\mathrm{N}$ increased highly significantly total tuber number per hill from 8.44 to 9.84. [23] also indicated that the number of tubers initiated by a particular cultivar could be influenced by the growing condition. [32] observed a considerable variation in tuber number among four cultivars. The report of [33] mentioned increasing application of blended fertilizers from 100\% NPSB to 200\% NPSB with N adjustment increased mean tuber weight of potato crop by about $11 \%$ and $16 \%$, respectively over the control treatment. The present study is in agreement with the finding of [31] application of $\mathrm{N}$ and P significantly increased average tuber weight (ATW). The report of [30] pointed out increasing phosphorous rate resulted in increases in marketable tuber numbers, total tuber numbers, and average tuber weight. Similarly, [8] reported that potato planted in the highest rate of blended NPSZnB fertilizer (199 kg ha-1) recorded the highest average tuber weight.

According to the report of [21], the increment of average tuber weight is in response to the increased supply of blended NPSB fertilizer might be due to more fast growth, more foliage and increase in leaf area and higher supply of 
phosphorous-containing fertilizer, which may have induced formation of bigger tubers thereby resulting in higher average tuber weight. Boron does have a direct influence on yield or related attributes as it plays a great role in root development through which, it facilitates more nutrient-up take and more stolen development used to produce more tubers. This result is in agreement with [34] in which B fertilization significantly increased tuber number and yield, as well as Boron-up take in addition to available nitrogen and Boron in the soil after harvest. Boron plays role in tuber bulking through reverting plant vegetative growth to root growth by hormonal control and facilitating carbohydrate transport to root (https://levitycropscience.com/b-is-for-bulking-using-boron-to-increase-potato-yield/). Boron stabilizes calcium in the cell wall and acts in synergy with calcium to improve plant resistance to disease, pest, and environmental stresses [35]. Boron was reported as increasing tuber yield applied with sulfur [36]. In agreement with the finding of [21] the application of blended NPSB fertilizer increased the weight of tuber in all potato varieties as compared to growing of varieties without fertilizer application (control). In line with the finding, [22] variety Belete had about 30.3 and 29.75\% higher tuber weight than Bubu. The present study is in agreement with the idea of different researchers like [37, 23, 38] yield differences among genotypes were attributed both by the inherent yield potential of genotypes and the growing environment as well as the interaction of genotype $\mathrm{x}$ environment.

The present study is in line with the finding of [31] increasing the level of applied $\mathrm{N}$ from 0 to $165 \mathrm{~kg} \mathrm{~N} / \mathrm{ha}$, increased total tuber yield by $94.14 \%$. Similarly, the finding of [27] indicated that increasing the rate of nitrogen increase total tuber yield per plot, average tuber weight, marketable tuber weight, unmarketable tuber weight, total tuber number/plant, and small tuber size.

[30] also reported that as Phosphorous rate increases marketable tubers, total tuber numbers, average tuber weight were highly increased as result of responsiveness of potato as compared to other tuber crops. Similarly, [39] reported that the interaction of nitrogen and phosphorous were significantly affected the marketable tuber. According to the report of [8] increasing blended NPSZnB fertilizer rate from 0 to $199 \mathrm{~kg} /$ ha increased marketable tuber number from 4.16 to 5.89/hill with non significant effect on unmarketable tuber number. In agreement with these, the report of [26] increasing NPS application rates marketable tuber yield of potato was highly significantly affected the main effect of NPS rate and potato variety.

\section{Conclusion}

This study revealed that there was a significant difference among the yields and yield components due to fertilizer rates. There was an interaction effect due to fertilizer rates and variety for any parameter considered. From this, it can be concluded that the fertilizer rates of NPSB + urea highly significantly affected the yield and yield component of potato under irrigation. Therefore, it is better to apply $300 \mathrm{~kg} / \mathrm{ha}$ NPSB $+150 \mathrm{~kg} / \mathrm{ha}$ urea to the Belete potato variety and $150 \mathrm{~kg} / \mathrm{ha}$ NPSB $+250 \mathrm{~kg} / \mathrm{ha}$ urea to Gudenie potato variety for high yield and high economic return in Holetta and areas with similar soe il type and agro-ecologies under irrigation for both Belete and Gudenie.

\section{References}

[1] CSA. (2017). Agricultural Sample Survey Report on Area and Production (Private Peasant Holdings Meher Season. Central Statistical Agency of Ethiopia, Statistical Bulletin).

[2] Tewodros, A., Paul, C. Struik, and Adane, H. (2014). Characterization of seed potato (Solanum tuberosum L.) Storage, pre-planting treatment and marketing systems in Ethiopia: the case of west-Arsi zone. African Journal of Agricultural Research, 9(15): 1218-1226.

[3] Girma, A. (2001). Influence of nitrogen and phosphorus on yield, yield components and tuber quality of two potatoes on Nitosols in Bako area. An MSc thesis presented to School of Graduate Studies of Alemaya University. P. 112.

[4] MoARD. (2005). (Ministry of Agriculture and Rural Development) Crop variety register. Crop development department, Issue No. 8. April 2005, Addis Ababa Ethiopia. P. 170.

[5] Berga Lemaga, Gebremedhin W/Georgis, Terrisa Jalleta, and Bereke-Tsehai Tuku. (1994). Potato Agronomy Research. In: Edward Herath and Lemma Dessalegn (Eds.). Proceedings of the Second National Horticultural Workshop of Ethiopia. Addis Ababa, 1-3 December 1992. Institute of Agricultural Research and Food and Agriculture Organization, Addis Ababa, Ethiopia.

[6] André, D., Peter, K., and Oscar O. (2014). Potatoes for Sustainable Global Food Security European Association for Potato Research, 57(3-4): 185-199.

[7] EthioSIS. (2016). The role of DSM in transforming agriculture: The case of Ethiopian soil information system. P. 35.

[8] Bewuket Getachew Bekele. (2018). Effect of Blended Npsznb Fertilizer and Cattle Manure Rates on Growth, Yield and Quality of Potato (Solanum Tuberosuml.) At Banja District, Awi Zone, North Western Ethiopia (Doctoral Dissertation, Jimma University).

[9] Kumar, R., and J. Singh. (2003). Regional water management modeling for decision support in irrigated agriculture. J. Irrig. Drain. Eng. ASCE., 129: 432-439. 
[10] Holzapfel, E. A., Leiva, C., Mariño, M. A., Paredes, J., Arumí, J. L., and Billib, M. (2010). Furrow irrigation management and design criteria using efficiency parameters and simulation models. Chilean Journal of Agricultural Research, 70(2010), No. 2, 70(2), pp. 287-296.

[11] Walker, W. R. and G. V. Skogerboe. (1987). Surface irrigation, Theory and practice. P. 386. Prentice-Hall, Englewood Cliffs, New Jersey, USA.

[12] Agricultural Transformation Agency (ATA). (2013). Status of soil resources in Ethiopia and priorities for sustainable management. Ethiopian agricultural transformation agency In: Global Soil partnership (GSP) for eastern and southern Africa. March 25-27, 2013, Nairobi, Kenya.

[13] EARO (Ethiopian Agricultural Research Organization). (2004).

[14] SAS (Statistical Analysis System) Software. (2009). Version 9.2. Inc. Carry, North Carolina, USA.

[15] CIMMYT. (1988). From agronomic data to farmer recommendations: An economics training. Retrieved from https://libcatalog.cimmyt.org/Download/cim/13803.pdf.

[16] EthioSIS (Ethiopian Soil Information System). (2014). Soil Fertility and Fertilizer recommendation Atlas of Tigray Region. Ministry of Agriculture (MoA) and Agricultural Transformation Agency (ATA).

[17] Sahlemdhin Sertsu. (1999). Draft guideline for regional soil testing laboratory. NFIA, Addis Ababa, Ethiopia.

[18] FAO (FOOD AND AGRICULTURE ORGANIZATION). (2006). Plant nutrition for food security .A guide for integrated nutrient management, Rome, 2006.

[19] MoARD (Ministry of Agriculture and Rural Development). (2011). Animal and plant health regulatory directorate. Crop variety register issue No. 12. Addis Ababa, Ethiopia.

[20] Tekalign Tadesse. (1991). Soil, plant, water, fertilizer, animal manure and compost analysis. Working Document No 13. International Livestock Research Center for Africa (ILCA), Addis Ababa. Kjedahl Jackson, M.L. 1958.

[21] Muluneh Siraj. (2018). Effects of Blended NPSB Fertilizer Rates on Growth, Yield and Yield Related Traits of Potato (Solanum tuberosum L.) varieties under irrigation.

[22] Getachew khasay. (2016). Response of potato (solanum tuberosum l.) Varieties to nitrogen and blended fertilizers under irigation at maichew, southern Tigray, Ethiopia: pp. 35-59.

[23] Elfinesh Firdissa. (2008). Processing quality of improved potato (solanum tuberosum l.) Varieties as influenced by growing environment, genotype and blanching Thesis. Haramaya University, Ethiopia: pp. 27-31.

[24] Lynch, D. R. and G. C. Tai. (1989). Yield and yield components of eight potato genotypes to water stress. Crop Sci. 29: 1207-1211.

[25] Morena, D. L., I. A. Guillen, and L. F. Garcia. (1994). Yield development in potato as influenced by cultivars and the timing and level of nitrogen fertilizer. Am. Potato J., 71: 165-171.

[26] Melkamu Alemayehu and Miniweyilet Jembere. (2018). Optimum rates of NPS fertilizer application for economically profitable production of potato varieties. Cogent Food and agriculture, (2018), 4: 1439663.

[27] Fayera. (2017). Yield and yield components of potato (Solanum tuberosum L.) as influenced by planting density and rate of nitrogen application at Holeta, West Oromia Region of Ethiopia. African Journal of Agricultural Research, 12, $2242-2254$. doi:10.5897/AJAR.

[28] Firew Gebremariam, Nigussie Dechassa, and Wassu Muhamed. (2016). Response of potato (solanum tuberosum L.) to the application of mineral nitrogen and phosphorus under irrigation in Dire Dawa, Eastern Ethiopia. Journal of natural sciences research. ISSN 2224-3186 (Paper), Vol. 6, No. 7, 2016.

[29] Sunil Kumar Yadav, G. K. Singh, Vikas Kumar Jain, and Anupam Tiwari. (2017). Response of potato (solanum tuberosum L.) Cultivars to different levels of nitrogen. International Journal of Current Microbiology and Applied Sciences ISSN: $2319-7706$ Volume 6 Number 8 (2017), pp. 2734-2739. Indian Potato Association, Shimla, 2, 889-899.

[30] Girma, Ch., Abebe, C., and Zeleke, O. (2017). Response of applied phosphorus fertilizer rate and plant spacing for potato (Solanum tuberosum L.) production on nitisols in central highland of Ethiopia. Greener Journal of Agricultural Sciences, 7, 255-262. doi: 10.15580/GJAS.

[31] Mulubrhan Haile. (2004). The effect of N, P, K fertilization on yield and yield Components of potato (Solanum tuberosum L.) grown on vertisols of Mekelle Area, Ethiopia. M.Sc. Thesis, the University of Haremaya, p. 15.

[32] Tekalign, T. and P. S. Hammes. (2005). Growth and productivity of potato as influenced by cultivar and reproductive growth II, Growth analysis, tuber yield and quality. Scientia Horticulturae. 105: 13-44.

[33] Desta Bekele. (2018). Evaluation of Blended and Non-blended Fertilizer Types and Rates on Potato (Solanum tuberosum L.) Yield and Yield Components at Assosa, Western Ethiopia, pp: 23-50.

[34] Sarkar, S., Banerjee, H., Ray, K., and Ghosh, D. (2018). Boron fertilization effects in processing grade potato on an Inceptisol of West Bengal, India. Journal of Plant Nutrition, 41(11), 1456-1470.

[35] Abdulnour, J. E., Donnelly, D. J., and Barthakur, N. N. (2000). The effect of boron on calcium uptake and growth in micropro- 
pagated potato plantlets. Potato Research, 43(3), 287-295

[36] Bari, M. S., M. G. Rabbani, M. S. Rahman, M. J. Islam, and A. T. Hoque. (2001). Effect of zinc, boron, sulphur and magnesium on the growth and yield of potato. Pakistan Journal of Biological Science, 4(9): 1090-1093. African Journal of Plant Science, 3(2): 016-024.

[37] Amamaw Yeshanew. (2007). Postharvest quality of potato (solanum tuberosum l.) cultivars as influenced by growing environment and storage period. Pp. 25-30.

[38] Habtamu Gebreselassie, Wassu Mohammed, and Beneberu Shimelis. (2016). Evaluation of potato (Solanum tuberosum L.) varieties for yield and yield components in Eastern Ethiopia. Journal of Biology, Agriculture and Healthcare, 6(5): 146-154.

[39] Burtukan Belachew. (2016). Effect of nitrogen and phosphorus rates on growth, yield, yield components and quality of potato ( Solunum tuberosum L). 